\title{
Thermal photons at PHENIX experiment
}

\author{
Veronica Canoa Roman ${ }^{1,2, \star}$ \\ ${ }^{1}$ for the PHENIX Collaboration \\ ${ }^{2}$ Stony Brook University
}

\begin{abstract}
Direct photons are a unique probe to study the properties of the medium created in heavy ion collisions. In particular low $\mathrm{p}_{\mathrm{T}}$ direct photons are of great importance since one expects that they are predominantly of thermal origin. In A+A systems PHENIX has observed a large yield of low $\mathrm{p}_{\mathrm{T}}$ direct photon that are emitted with a significant azimuthal anisotropy with respect to the reaction plane (v2). The mechanism responsible for the large yield and large v2 is not understood yet. Following recent evidence for collective behavior of charged particle production from small systems like $\mathrm{p}+\mathrm{A}, \mathrm{d}+\mathrm{Au}$, and $3 \mathrm{He}+\mathrm{Au}$, PHENIX has made systematic measurements of direct photons with different collision energies and system configurations. It has been found that the low $\mathrm{p}_{\mathrm{T}}$ direct photon yield $\mathrm{dN}_{\gamma} / \mathrm{d} \eta$ is proportional to $\left(\mathrm{dN}_{\mathrm{ch}} / \mathrm{d} \eta\right)^{\alpha}$. This behavior holds for beam energies measured both at RHIC and at the LHC in large-on-large systems, while data from small systems suggest an onset of QGP formation at low $\mathrm{dN}_{\mathrm{ch}} / \mathrm{d} \eta$. In this talk, I will report recent measurements of thermal photon and related observables.
\end{abstract}

\section{Introduction}

Direct photons are one of the probes to study the properties of a hot and dense QCD medium. Since photons do not interact strongly in heavy ion collisions, they can carry primordial information about the collision from the time of their production. Photons are very abundant in heavy ion collisions. However, the measurement of direct photons is challenging becuase most of them come from hadronic decays, mainly $\pi^{0}$ and $\eta$. The so-called direct photons can be determined experimentally by subtracting from the total yield the expected fraction coming from hadron decays. The primary photon contributor in the low $\mathrm{p}_{\mathrm{T}}$ region below $3 \mathrm{GeV} / \mathrm{c}$ is the thermal photons from the medium that keep the thermodynamic information from when they are generated. The contribution of these photons to the yield have an exponential shape, in analogy to the black body radiation. Meanwhile the contribution of the photon coming from initial states, usually call hard photons, have a shape following $1 / p_{\mathrm{T}}^{n}$. In contrast to the thermal photons, the contribuition of these photons start to be dominant at high $\mathrm{p}_{\mathrm{T}}(5$ $\mathrm{GeV})$.

At the PHENIX experiment, it was measured not only the direct photon yield but also the flow in $\mathrm{Au}-\mathrm{Au}$ collisions at $200 \mathrm{GeV}$. It was found a large yield and a large $v_{2}$. It has brought challenges to the theoretical models. Because a large yield implies early emission when the temperature is higher, but a large $v_{2}$ implies late emission when the flow is fully developed. The details can be found in [1].

^e-mail: vcanoaroman@gmail.com 

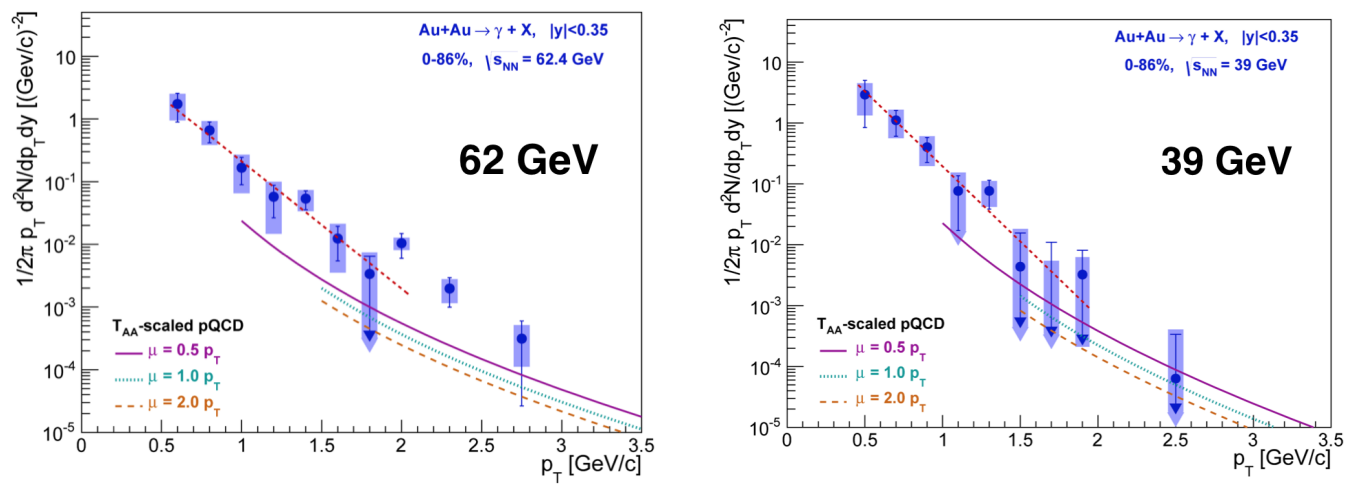

Figure 1. Low $p_{T}$ direct photon yield for $\mathrm{Au}+\mathrm{Au}$ collisions at $\sqrt{s_{N N}}=62.4 \mathrm{GeV}$ (left) and $\mathrm{Au}+\mathrm{Au}$ collisions at $\sqrt{s_{N N}}=39 \mathrm{GeV}$ (right).

\section{Direct photons in large systems}

The measurement of the direct photon yield in $\mathrm{Au}+\mathrm{Au}$ collisions at $200 \mathrm{GeV}$ is well established [2]. Recently PHENIX accomplished low momentum direct photon measurements in Au+Au at 62.4 $\mathrm{GeV}$ and $39 \mathrm{GeV}$ with the external conversion method [5]. In the external conversion method, the photons are measured through their conversions to electron-positron pairs at the HBD in the PHENIX detector system, and the fraction of direct photons is determined after tagging photons from neutral pion decays. Comparing the data with scaled pQCD calculations to Ncoll, we can find a clear excess over the scaled $\mathrm{p}+\mathrm{p}$ yield in the low $\mathrm{p}_{\mathrm{T}}$ region, as it has been seen in $\mathrm{A}+\mathrm{Au}$ at $200 \mathrm{GeV}$. The results can be seen in Figure 1.

$\mathrm{Cu}+\mathrm{Cu}$ collisions at $200 \mathrm{GeV}$ were also explored [3] via the internal conversion method. A clear direct photon signal excess was found at low $\mathrm{p}_{\mathrm{T}}$ region, when we compare with the scaled $\mathrm{p}+\mathrm{p}$ yield. The direct photon yield for $\mathrm{Cu}+\mathrm{Cu}$ minimum bias (MB) can be seen in the left panel of Figure 2. If we compare data from $\mathrm{Au}+\mathrm{Au} 40 \%-60 \%$ centrality which have a similar $\mathrm{Npart}$ as the $\mathrm{Cu}+\mathrm{Cu} 0 \%-40 \%$ centrality data, where the $\mathrm{Au}+\mathrm{Au}$ points are scaled by the Npart ratio $(66.4 / 56.0)$, we can find they are consistent within uncertainties, see Figure 2 in the right panel.

\section{Direct photons in small systems}

In $\mathrm{p}+\mathrm{p}$ and $\mathrm{p}+\mathrm{A}$ collisions at $200 \mathrm{GeV}$ collision, PHENIX has recently measured low momentum direct photons production with the external conversion method. While the $\mathrm{p}+\mathrm{Au} \mathrm{MB}$ direct photon signal is consistent with Ncoll $p+p$ scale, the most central $p+A u$ shows a hint of excess over the binary-scaled baseline, as seen in Figure 3. This non-zero excess yield in central $p+A u$ collisions is compatible with the production of QGP droplets in small central systems. The $\mathrm{p}+\mathrm{Au}$ result in MB is compatible with a previous measurement in $\mathrm{d}+\mathrm{Au} \mathrm{MB}$, where zero-excess was found [4].

\section{Direct photons scaling}

In order to compare the different collision systems and energies, and gain insight into the mechanism of the direct photon production, the yield can be integrated. It can be represented using the number 


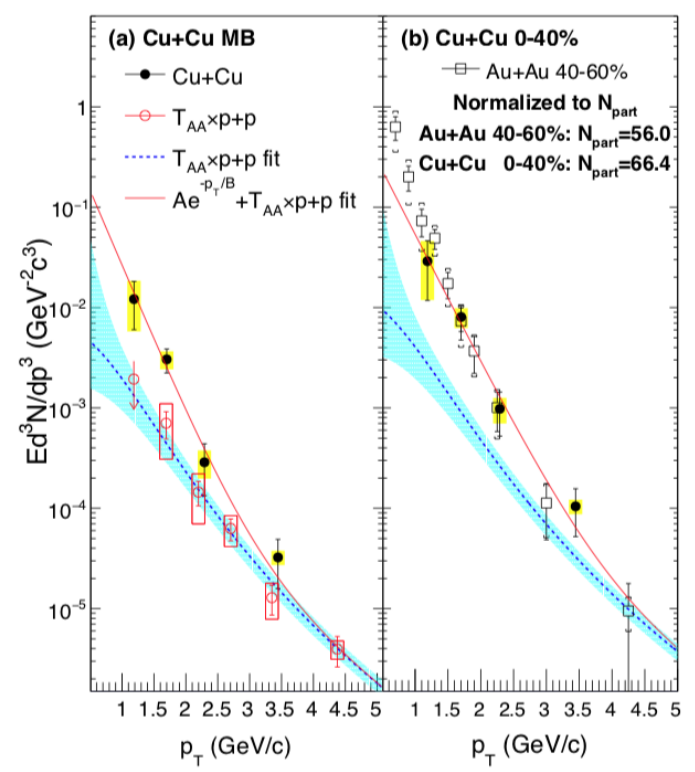

Figure 2. Low $p_{T}$ direct photon yield for $\mathrm{Cu}+\mathrm{Cu} \mathrm{MB}$ case at $\sqrt{s_{N N}}=200 \mathrm{GeV}$ (left), and $\mathrm{Cu}+\mathrm{Cu} 0 \%-40 \%$ centrality (black circles) and $\mathrm{Au}+\mathrm{Au}$ at $\sqrt{s_{N N}}=200 \mathrm{GeV}$ in similar number of participants centrality(open squares) (right).

of participants, $N_{\text {part }}$, or the number of binary collisions, $N_{\text {coll }}$. However, this is not useful to compare data at different energies. We can use instead charged particle multiplicity $\left(d N_{c h} / d \eta\right)$. It was found a interesting scaling behavior between Ncoll and $\left(d N_{c h} / d \eta\right)$ shown in Figure 4. The $N_{\text {coll }}$ scales like $\left(d N_{c h} / d \eta\right)^{\alpha}$, for all the energies, with $\alpha=1.25$ [5]. In order to quantify the direct photon spectra, and have a picture with different collision systems and energies together, we can integrate the invariant yield over some $p_{T}$ threshold value. Figure 5 shows the integrate direct photon spectra for $p_{T}>1$ $\mathrm{GeV} / \mathrm{c}$ (left) and $p_{T}>5 \mathrm{GeV} / \mathrm{c}$ (right).

In the plot, we put together all the data for direct photons at PHENIX and also data for ALICE [6]. We can observe a Universal scaling behavior of $d N_{\gamma} / d \eta$ with $\left(d N_{c h} / d \eta\right)^{1.25}$, independent of the system, energy or centrality at low $p_{T}$ direct photons for large systems. In the low multiplicity region one can see the gradually increasing trend of the integrated yield of the small systems, which seems to intersect with the trend from the large systems. The integrated yield at high $p_{T}, p_{T}>5 \mathrm{GeV} / \mathrm{c}$, follows the $\mathrm{p}+\mathrm{p}$ fit scaling by the number of collisions for $\mathrm{Au}+\mathrm{Au}$ data as expected, where the dominant photons are the ones that come from hard scattering.

\section{Conclusions}

The PHENIX collaboration has performance measurement at low momentum direct photon in $\mathrm{Au}+\mathrm{Au}$ collisions at $200 \mathrm{GeV}, 62 \mathrm{GeV}$ and $39 \mathrm{GeV}, \mathrm{Cu}+\mathrm{Cu}$ collisions at $200 \mathrm{GeV}$, and p+Au at $200 \mathrm{GeV}$. When we put all the data from the large systems together, it was found a surprising scaling behavior of direct photons in large systems. At low $p_{T}$ the direct photons integrated yield follows a universal scaling as a function of the charged particle multiplicity $\left(d N_{c h} / d \eta\right)^{\alpha}$, with $\alpha=1.25$. The scaling may be an indication that direct photons are produced during the transition from QGP to the hadron 

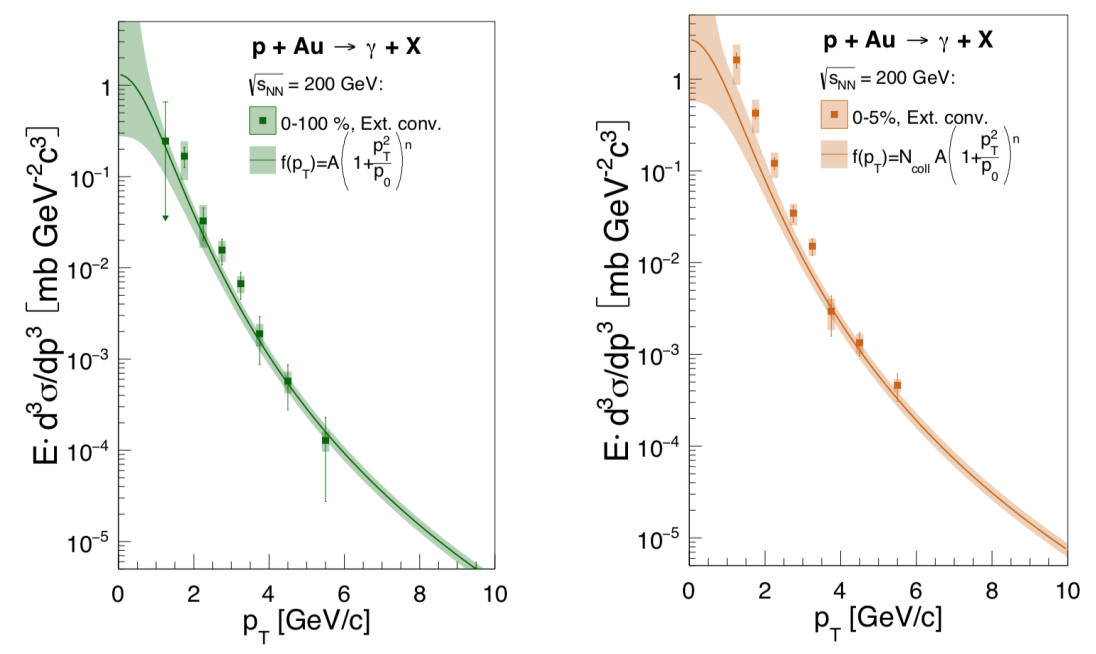

Figure 3. Low $p_{T}$ direct photon yield in $\mathrm{p}+\mathrm{Au} \mathrm{MB}(\mathrm{left})$ and $\mathrm{p}+\mathrm{Au}$ central collision(right) at $\sqrt{s_{N N}}=200 \mathrm{GeV}$.

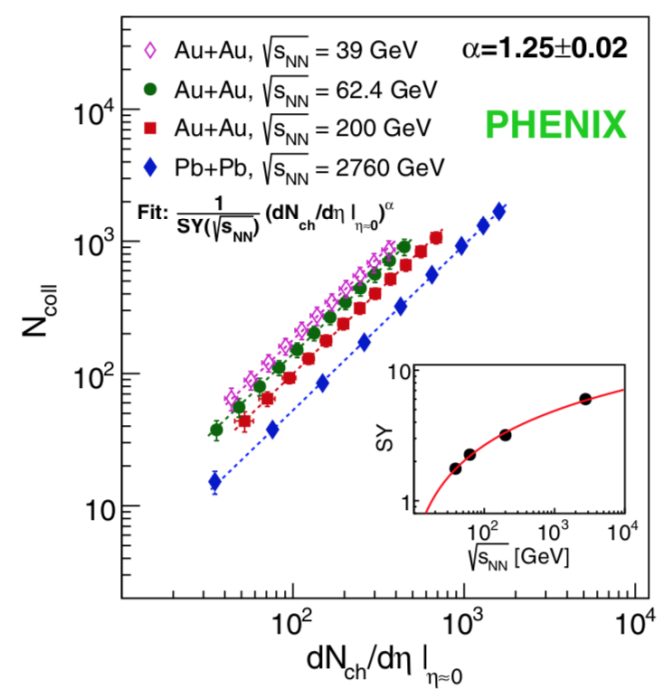

Figure 4. $N_{\text {coll }}$ vs. charged-particles multiplicity $d N_{c h} / d \eta$ for four beam energies. More details in [5].

gas. The non-zero excess over the scaled $\mathrm{p}+\mathrm{p}$ yield in central $\mathrm{p}+\mathrm{Au}$ collisions can indicate a possible formation of QGP droplets in the small systems.

\section{References}

[1] A. Adare et al. (PHENIX Collaboration), Phys.Rev. C 94, 064901 (2016). 

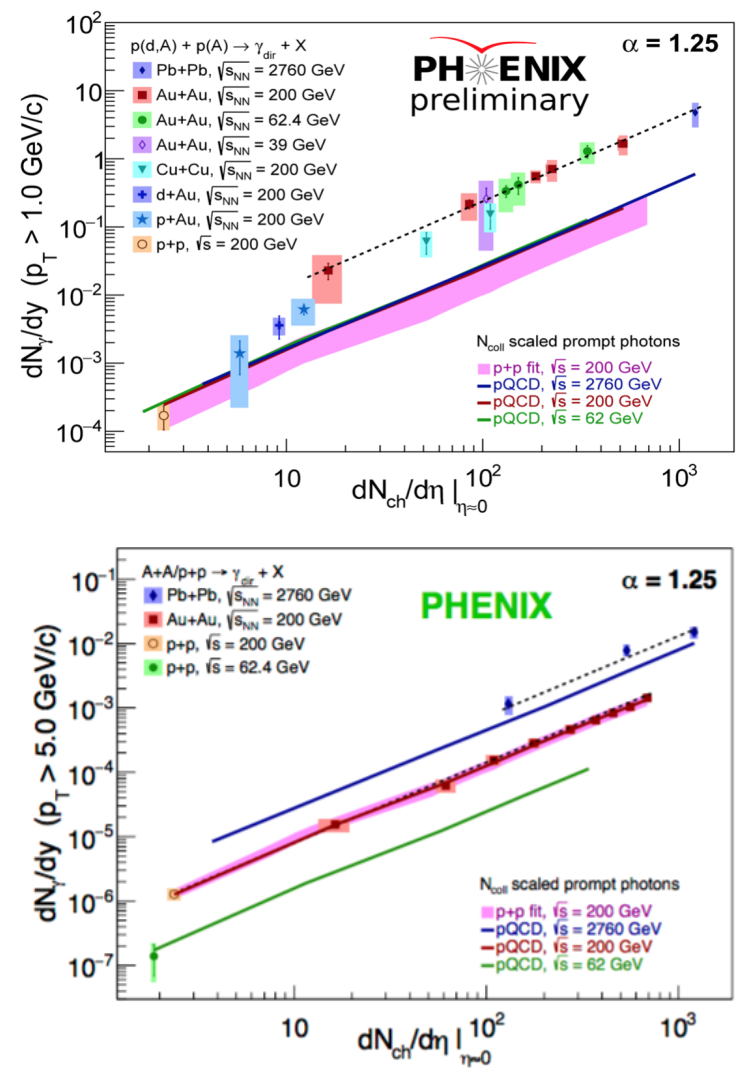

Figure 5. Integrated direct photon yield for different collision systems and energies vs $d N_{c h} / d \eta$, integrated in $p_{T}>1 \mathrm{GeV} / \mathrm{c}$ (top) and $p_{T}>5 \mathrm{GeV} / \mathrm{c}$ (bottom)[5].

[2] A. Adare et al. (PHENIX Collaboration), Phys.Rev. C 91, 064904 (2015).

[3] A. Adare et al. (PHENIX Collaboration), Phys.Rev. C 98, 054902 (2018).

[4] A. Adare et al. (PHENIX Collaboration), Phys.Rev. C 87, 054907 (2013).

[5] A. Adare et al. (PHENIX Collaboration), Phys.Rev.Lett. 123, 022301 (2019).

[6] J. Adam et al. (ALICE Collaboration), Phys.Lett.B 754, 235 (2016). 